Provided for non-commercial research and educational use only. Not for reproduction or distribution or commercial use.

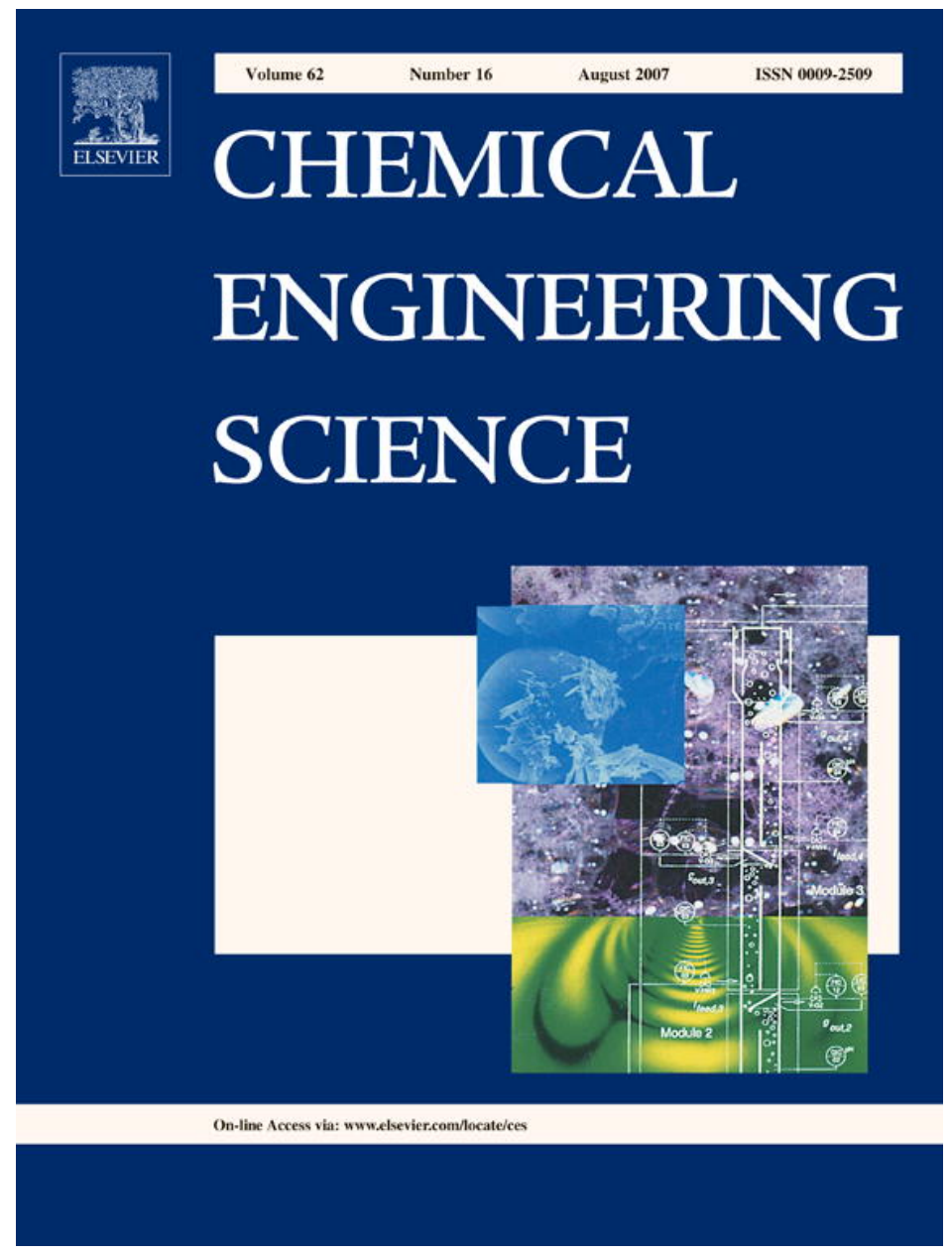

This article was published in an Elsevier journal. The attached copy

is furnished to the author for non-commercial research and education use, including for instruction at the author's institution, sharing with colleagues and providing to institution administration.

Other uses, including reproduction and distribution, or selling or licensing copies, or posting to personal, institutional or third party websites are prohibited.

In most cases authors are permitted to post their version of the article (e.g. in Word or Tex form) to their personal website or institutional repository. Authors requiring further information regarding Elsevier's archiving and manuscript policies are encouraged to visit: 


\title{
NMR microimaging of fluid flow in model string-type reactors
}

\author{
Igor V. Koptyuga ${ }^{\mathrm{a}, *}$, Kirill V. Kovtunov ${ }^{\mathrm{a}}$, Edo Gerkema ${ }^{\mathrm{b}}$, Lioubov Kiwi-Minsker ${ }^{\mathrm{c}}$, \\ Renad Z. Sagdeev ${ }^{\mathrm{a}}$ \\ anternational Tomography Center, 3A Institutskaya St., Novosibirsk 630090, Russia \\ ${ }^{\mathrm{b}}$ Laboratory of Biophysics and Wageningen NMR Centre, Wageningen University, Dreijenlaan 3, 6703 HA Wageningen, The Netherlands \\ ${ }^{\mathrm{c}}$ Group de Génie de la Réaction Catalytique (GGRC), SB-ISIC, Station 6, Ecole Polytechnique Fédérale de Lausanne (EPFL), CH-1015 Lausanne, Switzerland
}

Received 25 December 2006; received in revised form 26 April 2007; accepted 28 April 2007

Available online 22 May 2007

\begin{abstract}
Magnetic resonance microimaging (MRM) was employed to obtain quantitative velocity maps of water flowing in the channels possessing unconventional cross-section shapes formed by a bundle of parallel fibers within a tubular string-type reactor. The maps obtained demonstrate the presence of large amounts of an almost stagnant liquid in the stretched corners of the cross-sections representing distorted triangles or squares. This fact together with the irregularity of the filaments packing in the model string-type reactor was demonstrated to lead to a broad residence time distributions (RTDs) for liquid flow. Next, the pulsed field gradient NMR (PFG NMR) technique was employed to compare transport of water with that of butane gas in the same model string-type reactor. The experimentally measured average propagators (travel distance probability density functions) have demonstrated that Taylor dispersion can lead to much better RTDs for gas as compared to liquid in channels with sub-millimeter equivalent diameters. The PFG NMR data were compared with the RTD obtained using the conventional tracer time-of-flight transient response method. It is concluded that due to the differences in the quantities actually measured by the two techniques, and the significant differences in the measurement length scales (microns to $1-2 \mathrm{~cm}$ for NMR/MRM, tens of centimeters for transient response methods), there is no reliable way of directly comparing these results. The information obtained by NMR/MRM and more conventional techniques such as time-of-flight should be considered as complementary. In particular, NMR/MRM can reveal the reasons for the observed overall reactor performance by providing access to the transport processes on short length scales inside the reactor and by revealing structure-transport interrelations.
\end{abstract}

(C) 2007 Elsevier Ltd. All rights reserved.

Keywords: Imaging; Tomography; Laminar flow; Compact string reactor; Dispersion; Residence time distribution

\section{Introduction}

Microreaction technology has experienced spectacular developments in the recent past. Microstructured reactors with the sub-millimeter characteristic channel diameters are known to have a number of important advantages over conventional reactor types (Ehrfeld et al., 2000), including significantly larger surface-to-volume ratio and heat transfer coefficients. Despite the laminar character of flow, the influence of mass transfer on the reaction rate can be substantially reduced owing to very short transverse diffusion times. High rates of heat transfer allow very fast heating and cooling of reaction mixtures in open

\footnotetext{
* Corresponding author. Tel.: +7383 3333561; fax: +73833331399.

E-mail address: koptyug@tomo.nsc.ru (I.V. Koptyug).
}

reactor systems (Alépée et al., 2000), safe utilization of higher reaction temperatures, and significantly reduced reactor volumes. Catalytic reactions can be carried out with a reduced amount of catalysts and with short and well-defined residence times of reacting mixtures, avoiding unwanted consecutive reactions. In consequence, microreactors allow accurate control of chemical transformations leading to high efficiency and selectivity. Among other things, safe processing of toxic and explosive substances and accurate control of highly exo- or endothermic processes become possible.

When microreactors are used for heterogeneous catalytic processes, the main problems that arise are associated with the introduction of the catalyst and accommodation of the microreactor in the available technological lines (Kiwi-Minsker and Renken, 2005). A straightforward approach is to pack 

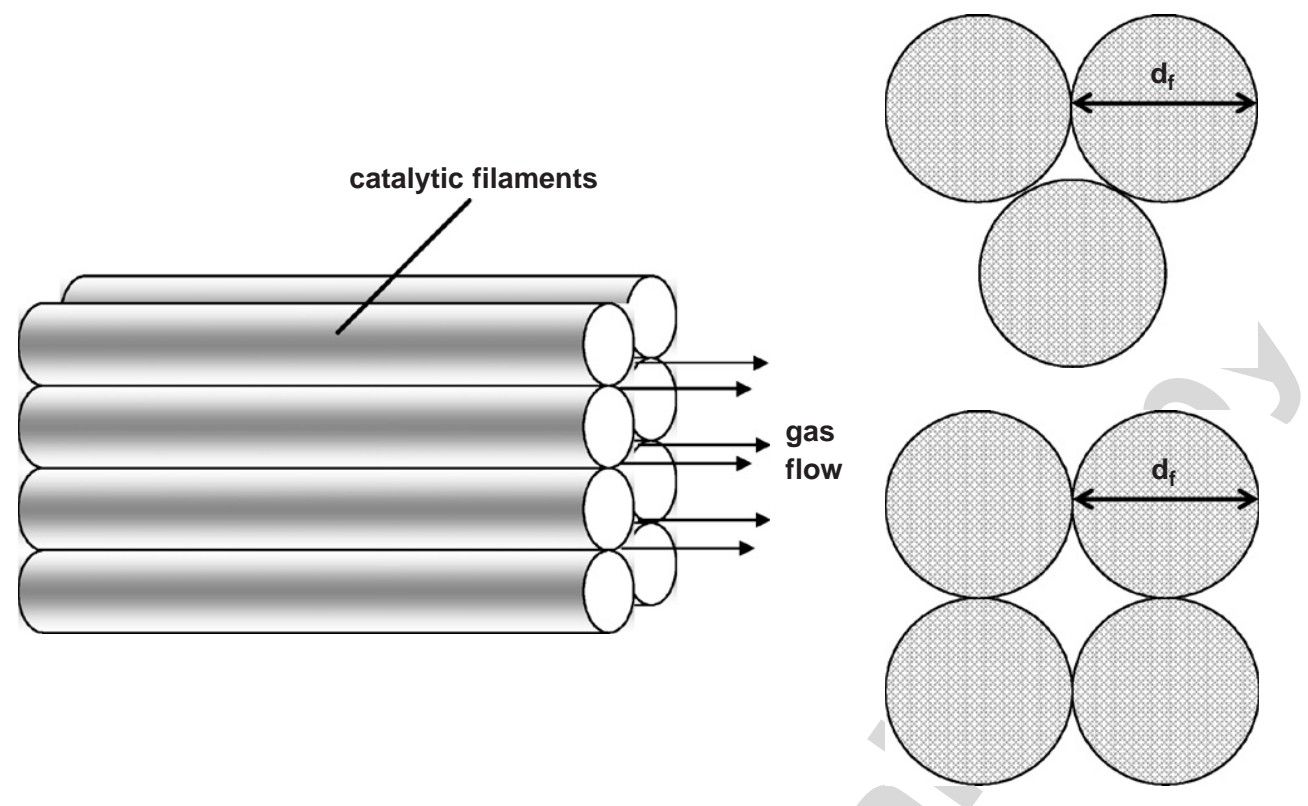

Fig. 1. Schematic representation of the channel structure for a bundle of filaments or strings. The equivalent channel diameters are $0.103 d_{\mathrm{f}}$ for the top geometry and $0.273 d_{\mathrm{f}}$ for the bottom one.

microchannels with a catalyst powder. The drawback of micropacked-bed reactors is the resulting high pressure drop. In addition, each channel must be packed identically to avoid a broad residence time distribution (RTD) in the reactor system. To circumvent the problems associated with the use of randomly packed beds, a novel compact string reactor was proposed recently (Wolfrath et al., 2001; Horny et al., 2004). The reactor consists of a bundle of catalytic filaments $10-500 \mu \mathrm{m}$ in diameter packed in a tube and aligned parallel to the reactor walls. The fluids flow in the interstitial channels formed by the filaments (Fig. 1). The equivalent hydraulic diameters of these channels, defined as four times the ratio of the channel cross-sectional area to its circumference, are in the range from dozens to a few hundred microns and ensure laminar axial flow and short transverse diffusion times. Therefore, this arrangement should give flow hydrodynamics similar to that in multi-channel microreactors known to have narrow RTDs.

The conventional approach often employed to study transport properties of various reactors is the tracer time-of-flight technique, which was used to characterize compact string reactors as well (Wolfrath et al., 2001; Horny et al., 2004). This approach can readily characterize the performance of a given reactor by means of evaluating its RTD. However, such measurement is performed at the reactor outlet and therefore reflects the transport properties averaged over the entire reactor. Therefore, no information is available about the reasons behind unsatisfactory transport properties of a reactor, and no guidance is provided which could help improve the observed RTD. At the same time, in string reactors discussed above and in other flow devices possessing similar structure (e.g., hemodialyzers), the fluid flows in multiple channels with cross-sections having a form of curvilinear triangles or squares with stretched corners (Fig. 1) or other unconventional shapes for irregular pack- ings. In such a case, the flow pattern can be revealed either by numerical calculations or experimental mapping of fluid flow. However, it can be expected that in practice the structure of a reactor may to a certain extent deviate from a regular packing. Therefore, experimental studies are essential for both revealing the actual structure and assessing the influence of structure properties and nonidealities on the fluid flow characteristics.

NMR imaging (MRI) and microimaging (MRM) and pulsed field gradient NMR (PFG NMR) techniques are now routinely applied for the structural studies of liquid containing objects and for evaluation of fluid transport properties in a variety of porous objects (Callaghan, 1991; Watson et al., 2002; Fukushima, 1999; Koptyug and Sagdeev, 2002) including packed beds (Gladden, 2003; Goetz et al., 2002; Gladden et al., 2002; Mansfield and Bencsik, 1998; Tessier et al., 1997; Lebon and Leblond, 2002). However, applications of these techniques to study flow of fluids in the microstructured reactors only begin to emerge (Hilty et al., 2005; Han et al., 2006). At the same time, MRM and PFG NMR can provide access to important information such as flow velocity maps, diffusion and dispersion coefficients, displacement distributions, etc. Therefore, application of these techniques to the evaluation of transport in microreactors appears to be highly promising and will, without doubt, attract much attention in the future. The possibility to apply MRI and PFG NMR measurements for the studies of liquid flow in fiber packs has been demonstrated for model and clinical hemodialyzer units (Laukemper-Ostendorf et al., 1998; Han and Blumich, 2000; Han and Stapf, 2006) and hollow membrane bioreactors (Heath et al., 1990). In this work, MRM and PFG NMR techniques are used to characterize flow of gases and liquids in model string-type reactors, and the results obtained are compared to the measurements performed by a conventional tracer time-of-flight technique. 


\section{Experimental}

\subsection{MRM and PFG NMR measurements}

Butane-propane gas liquefied in tanks for domestic burners was purchased from a commercial vendor. Distilled water was used in the water flow studies. For the studies of gas flow in a single capillary with a circular cross-section, glass pipette with $2.4 \mathrm{~mm}$ i.d. was used. Model string-type reactors were comprised of a large number of long plastic strings 1 or $0.7 \mathrm{~mm}$ in diameter packed in plastic cylinders $20 \mathrm{~cm}$ long with the inside diameters of $16 \mathrm{~mm}$. The strings were approximately parallel to the cylinder axis.

During the gas flow experiments, the gas was expanding from the tank into the supply tubing and was leaving the setup through the open end of the exhaust tubing into the fume hood. The flow of water was created by a thermostat pump. In all the experiments, the orientation of model reactors was vertical, and water or gas entered a reactor at the bottom and left at the top.

All NMR and MRI experiments were performed at $300.13 \mathrm{MHz}$ on a Bruker DRX 300 NMR spectrometer equipped with a vertical bore superconducting magnet and microimaging accessories. The spin-echo pulse sequence was employed in all flow imaging experiments (Callaghan, 1991; Seymour and Callaghan, 1997). The two-dimensional (2D) images were detected in the plane perpendicular to the cell axis, with $(75 \mu \mathrm{m}) \times(75 \mu \mathrm{m})$ spatial resolution in the image plane for a $1.5 \mathrm{~mm}$ thick slice. It took ca. $1 \mathrm{~h}$ to acquire each image, with two images required to calculate a $2 \mathrm{D}$ flow velocity map (Callaghan, 1991; Fukushima, 1999; Koptyug and Sagdeev, 2002). In all the experiments, the velocity component along the fibers (reactor) was measured. For the measurements of average propagators, a stimulated echo pulse sequence was used (Packer and Tessier, 1996; Lebon et al., 1996).

\subsection{RTD measurements}

The RTDs were measured in a tubular reactor $(230 \mathrm{~mm}$ long, $15 \mathrm{~mm}$ inside diameter) packed with irregular silica particles $(0.7-1.5 \mathrm{~mm})$ or glass beads $(100-160 \mu \mathrm{m}$ in diameter), or alternatively with the silica fibers in the form of threads. Each thread had a diameter of about $0.5 \mathrm{~mm}$ consisting of ca. 100 filaments each having a diameter of ca. $7 \mu \mathrm{m}$. The silica threads were placed in a tube parallel to each other and aligned along the reactor walls. The catalytic bed arranged in this manner had about 300 threads per square centimeter of the reactor crosssection giving the porosity of the bed of ca. 0.4. A similar packing of silica threads (200 mm long, $11 \mathrm{~mm}$ inside diameter) was used in the PFG NMR experiments.

The experimental setup for the RTD measurements consisted of the gas-feed section, the reactor, and the quadrupole mass spectrometer (QMS 400, Balzers, Liechtenstein). A fourway valve allowed switching between $\mathrm{N}_{2}$ or $\mathrm{Ar} / \mathrm{N}_{2}$ mixture (10 vol\%). Argon was used as the tracer and its concentration was determined by QMS every $30 \mathrm{~ms}$. The gases were supplied through mass flow controllers (Bronkhorst High-Tech B.V.).
RTD in catalytic bed was determined by measuring the response at the reactor outlet to a switch from $\mathrm{N}_{2}$ to $10 \% \mathrm{Ar}$ in $\mathrm{N}_{2}$ mixture at the reactor inlet.

\section{Results and discussion}

\subsection{MRM and PFG NMR studies}

As mentioned above, the cross-sections of the microchannels in a regularly packed string-type reactor can be expected to have a non-conventional shape of curvilinear triangles or squares with stretched corners (Fig. 1). Therefore, the first experiment was carried out to visualize fluid flow in such channels and to reveal its features in a model system imitating such geometry. To this end, three long rigid plastic cylinders $(5 \mathrm{~mm}$ outside diameter) were squeezed into a piece of PVC tubing parallel to the latter and to each other. The results obtained are shown in Fig. 2. The figure shows the contour map of velocity iso-lines which reveal the spatial organization of the flow of water in this geometry. In particular, the number of contour lines in the inner channel is smaller than in the channels adjacent to the walls of the outer boundary and indicates lower flow velocity for a channel with a smaller cross-sectional area. This fact is also apparent from the cross-section of the velocity map shown in Fig. 2c. In each channel, the outer contours have a shape of a rounded triangle while the inner contours converge to a circle. There is little flow in the long corners of triangles which thus form regions of an almost stagnant liquid. Note that one of the inner tubes got filled with the liquid and can serve as zero flow velocity reference since it is plugged at one end and thus carries no flow.

The results presented in Fig. 2 clearly show that such flow geometry can be very unfavorable for practical applications. The inter-cylinder channels and especially the regions adjacent to the walls of the outer tube are characterized by large volumes of liquid with low or almost zero flow velocities and will inevitably lead to very broad RTDs. Since the diameter of the "fibers" in this experiment is relatively large, the flow behavior of gases will be similar to that observed for liquids.

However, in systems of practical importance, such as microreactors, the channels are much narrower. We therefore have studied flow patterns in a model string-type reactor comprising a large number of flexible plastic strings packed in a rigid cylinder. Fig. 3 depicts flow maps obtained for two such structures with the strings $1 \mathrm{~mm}$ (a) and $0.7 \mathrm{~mm}$ (b) in diameter packed in a tube with $16 \mathrm{~mm}$ inside diameter. One of the advantages of MRM studies is that the flow information is obtained along with the structural information about the flow geometry and thus flow maps are directly linked to the structure under study. In particular, the maps in Fig. 3 demonstrate that the packing of strings is far from being perfect, with a somewhat better packing of smaller diameter strings (Fig. 3b) As a result, most of the flow proceeds through the channels with large cross-sectional area in those regions where the separation of strings is larger than average. At the same time, in some of such channels the flow velocities are very low. The likely reason is that the strings do not form perfectly straight channels for fluid flow. Therefore, 
a

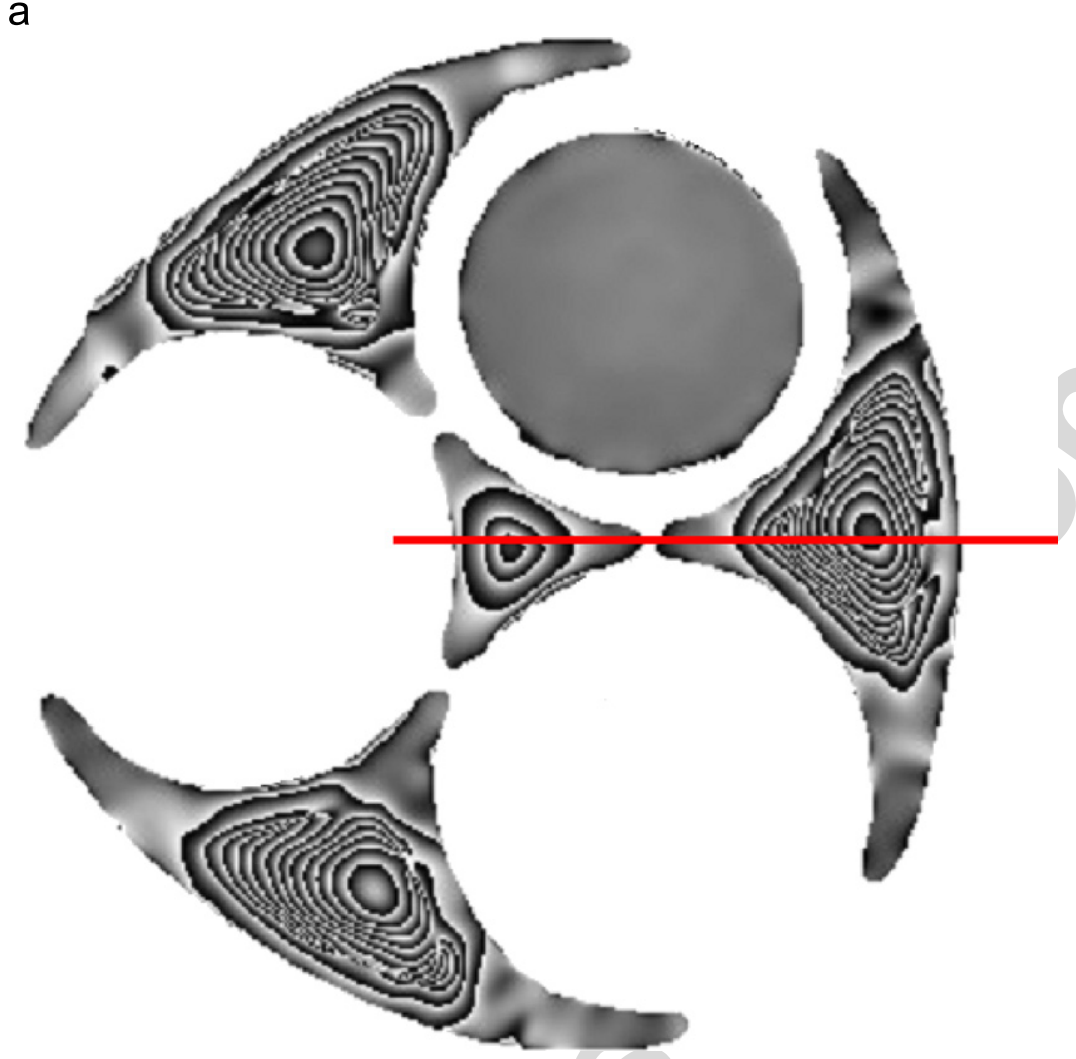

b

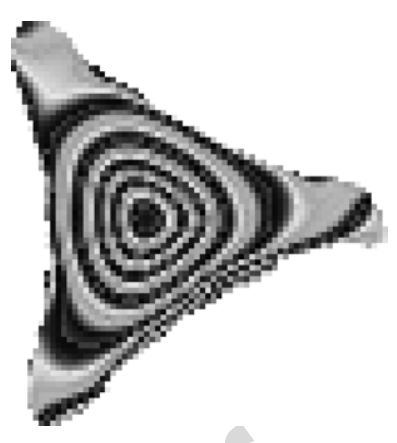

C

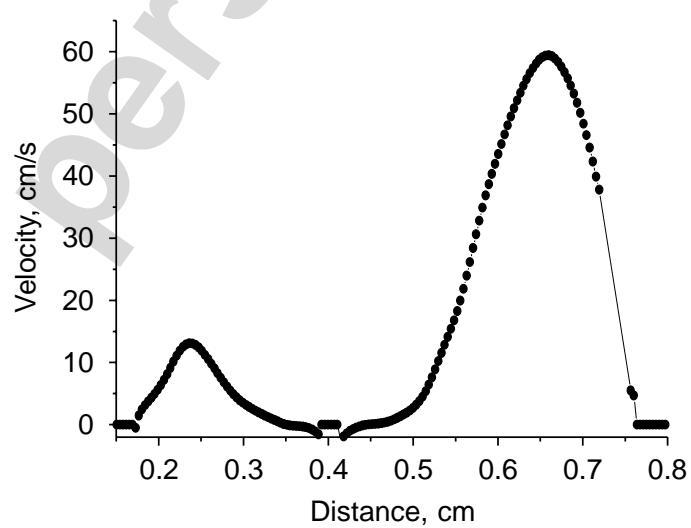

Fig. 2. Water flow velocity map for a model object comprised of three plastic tubes $5 \mathrm{~mm}$ in diameter inserted into a bigger PVC tube. Spatial resolution $150 \times 150(\mu \mathrm{m})^{2}$, digital resolution $38 \times 38(\mu \mathrm{m})^{2}$, thickness of the imaged slice $3 \mathrm{~mm}$. (a) Complete map showing flow velocity contour lines for the entire cross-section. (b) Central triangular channel shown with a larger number of velocity contour lines. (c) One-dimensional velocity profile along the horizontal line shown in (a).

the channel geometry changes along the reactor and can be quite different upstream or downstream of the imaged slice, affecting flow patterns in the slice selected for imaging (imaging slice thickness is $1.5 \mathrm{~mm}$ ). The channels where flow pattern closely resembles that of a triangular channel with concave sides are mostly located near the reactor walls where packing of the strings is more regular. At the same time, for liquid flow the resolution of MRM experiments $(10-40 \mu \mathrm{m})$ is sufficient to obtain complete flow velocity maps in the inter-string spaces.

It is easy to deduce from the maps shown in Fig. 3 that the RTD for liquid flow in such structures will be quite broad. In- deed, Fig. 4 shows the distribution (histogram) of flow velocities calculated from the flow velocity map shown in Fig. 3a. It is highly weighted toward low flow velocities, with a long tail stretching toward higher velocities. The histogram obtained from the flow map of Fig. $3 b$ (not shown) exhibits the same features. In this respect, the flow patterns observed in Figs. 2 and 3 are even worse than those of a laminar flow in a pipe. Such unfavorable behavior is caused by two factors. First, the broad distribution of channels cross-sections leads to a relatively large number of channels with relatively low average velocities. Next, in each channel the distribution of velocities is 
a

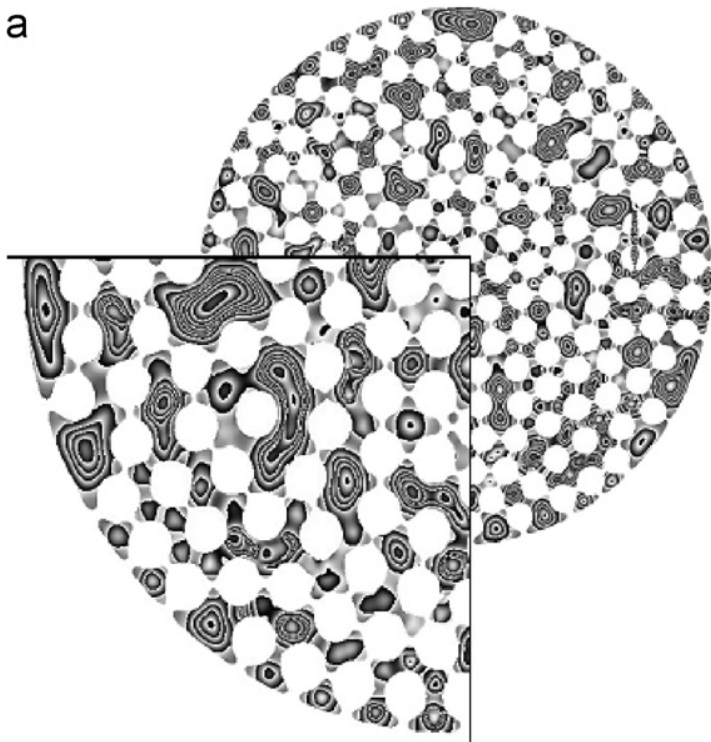

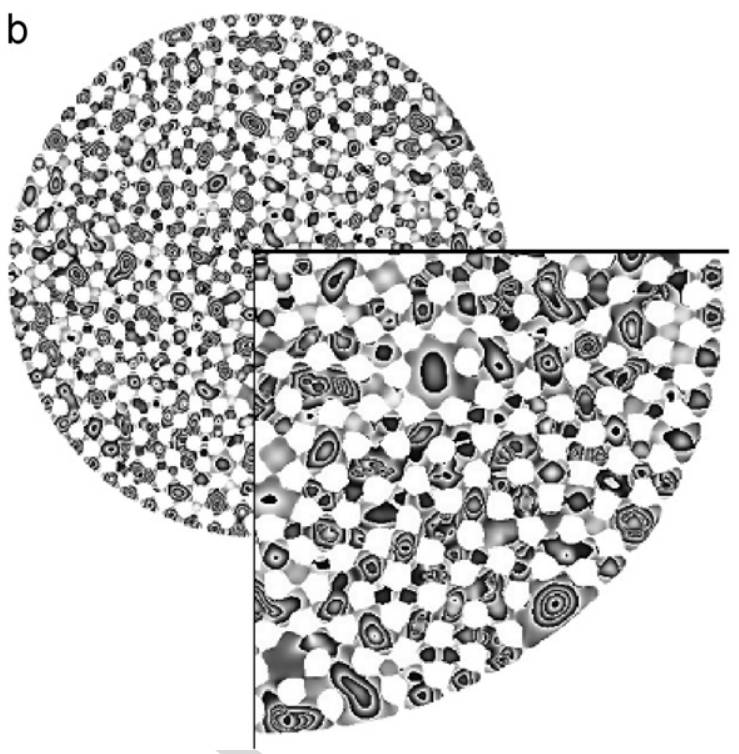

Fig. 3. Water flow velocity maps for the two model string-type reactors with (a) $1 \mathrm{~mm}$ and (b) $0.7 \mathrm{~mm}$ diameter strings demonstrating flow velocity contour lines for the respective geometries.

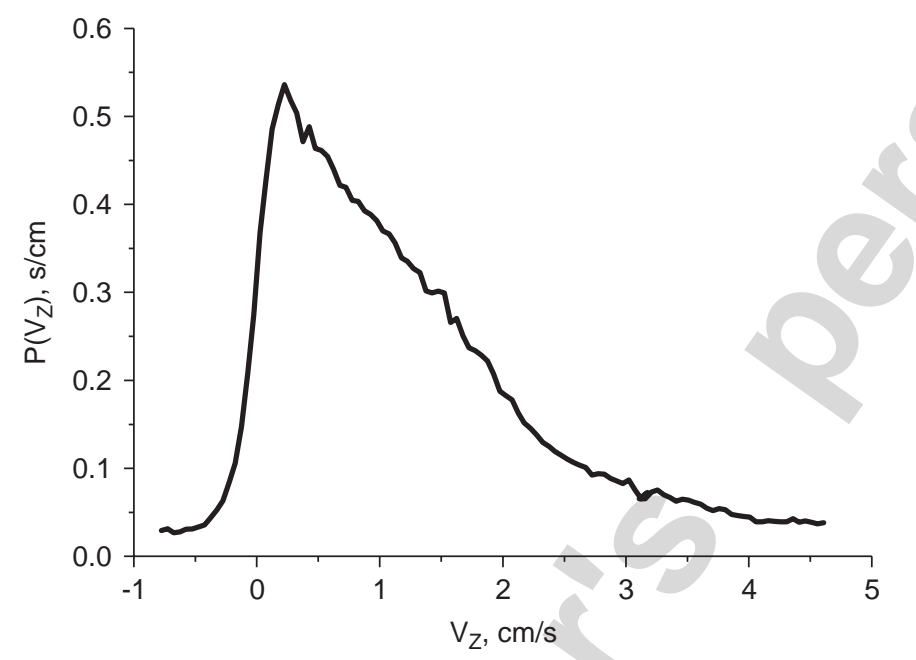

Fig. 4. Histogram of the distribution of velocities obtained by counting pixels of the flow velocity map shown in Fig. 3a.

weighted toward lower flow velocities because of the channel cross-section shape with stretched corners (cf. Figs. 1, 2, and 4). All this leads to a liquid flow velocity distribution which is very far from plug-flow behavior. Consequently, this flow pattern will not give narrow RTDs desirable in many practical applications.

In chromatographic columns and in packed-bed reactors, the instantaneous distribution of flow velocities is also weighted heavily toward lower flow velocities (Packer and Tessier, 1996; Lebon et al., 1996; Tallarek et al., 1998). Nevertheless, at observation times longer than the time required for molecules to travel over distances larger than the characteristic pore size (a few bead diameters for packed beds (Lebon et al., 1997), the distribution of displacements in the direction of main flow is usually centered at the average displacement ( $U \Delta$, where $U$ is the interstitial flow velocity and $\Delta$ is the encoding time) and has a Gaussian shape, i.e., is a good approximation of a plug flow. The reason is that molecule velocity is modulated as the molecule travels along a complex network of channels comprising the void space of a packed bed. This mechanism works for both liquids and gases, therefore similar transport characteristics can be expected for various fluids. This mechanism, however, does not operate at short length scales in flow geometries studied in this work because the channels are not tortuous and branched enough to substantially alter velocities of molecules as they travel along the reactor. In such a case, another mechanism of hydrodynamic dispersion can become efficient under certain conditions, namely the modulation of molecule velocity due to its diffusion across the stream lines (Taylor dispersion, Taylor, 1953). The efficiency of this mechanism will increase with the decrease of the channel cross-section and the increase in the diffusivity of the fluid. Since the molecular diffusion coefficients for gases are roughly 4 orders of magnitude higher compared to liquids, it is reasonable to assume that transport of gases in these string-type reactors can be quite different from the transport of liquids visualized above. At the same time, it will be impossible to use the approach utilized above (detection of flow velocity maps) to study gas flow patterns in such systems with sub-millimeter channels for gases with equilibrium polarization of nuclear spins (Koptyug et al., 2000, 2002). Therefore, a different experimental approach is required to compare the behavior of gases and liquids in narrow channels.

Fortunately, it is not necessary to detect complete flow velocity maps to obtain histograms like the one shown in Fig. 4. Such distributions can be obtained directly, in a PFG NMR experiment (Callaghan, 1991; Lebon and Leblond, 2002; Packer and Tessier, 1996). The latter encodes displacements of individual molecules in a chosen direction (e.g., $R_{Z}=Z-Z_{0}$ ) 


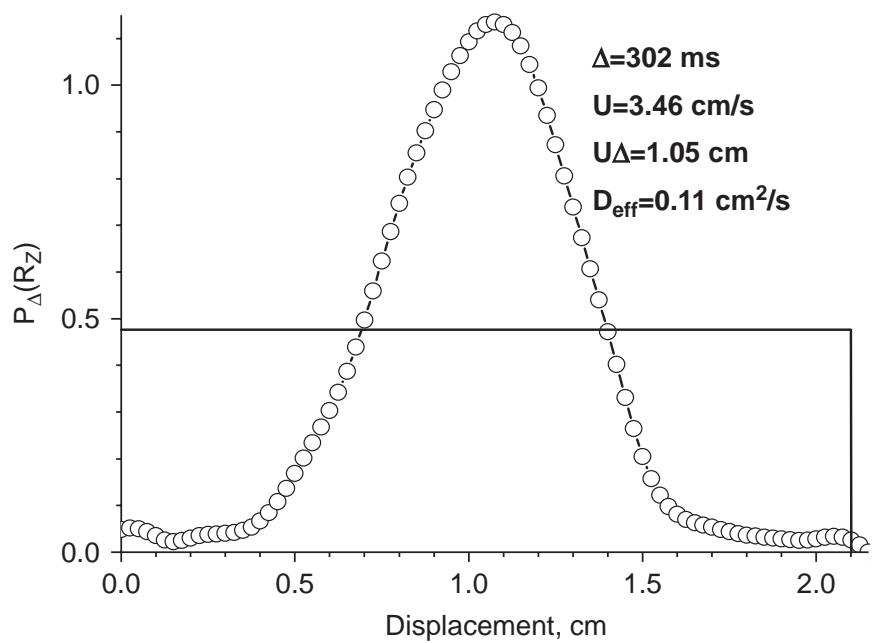

Fig. 5. Average propagator (displacement probability density) for butane gas flowing in the glass capillary with $2.4 \mathrm{~mm}$ inside diameter (circles). Solid line shows a top-hat "propagator" expected in the absence of fast transverse diffusion of the flowing fluid (i.e., without Taylor dispersion). The displacement encoding time was $\Delta=302 \mathrm{~ms}$.

for a preset encoding time $\left(\Delta=t-t_{0}\right)$. Such experiment directly yields the distribution of displacements $P_{\Delta}\left(R_{Z}\right)$, which is equivalent to a travel distance probability density function (Amin et al., 1997) and is often called an "average propagator" (Seymour and Callaghan, 1997). The dimensionless quantity $P_{\Delta}\left(R_{Z}\right) \mathrm{d} R_{Z}$ characterizes the probability for a molecule to have a displacement between $R_{Z}$ and $R_{Z}+\mathrm{d} R_{Z}$ for the given travel time $\Delta$. The direction of measured displacements corresponds to the direction of the applied magnetic field gradient and thus can be selected at one's will. The average propagator measured in a PFG NMR experiment, $P_{\Delta}\left(V_{Z}\right)=P_{\Delta}\left(R_{Z} / \Delta\right)$, is similar to the histogram of Fig. 4 , but there are some essential differences between the two. In particular, PFG NMR is sensitive not only to the convective flow, but also to diffusive displacements of molecules (Amin et al., 1997) which can be particularly important when studying transport of gases. As an example of the average propagator measurement, Fig. 5 demonstrates the distribution of displacements detected by PFG NMR for butane gas flowing in a single pipe with $2.4 \mathrm{~mm}$ inside diameter. It should be noted that for a fully developed laminar flow of a fluid (e.g., water) with insignificant transverse diffusion during the displacement encoding time of $300 \mathrm{~ms}$ used in the experiment, the expected distribution of displacements is a top-hat function shown with a solid line. Therefore, the experimentally detected propagator demonstrates the importance of Taylor dispersion for gas flow even in this relatively thick channel.

The results of the propagator measurements in the string reactors are presented in Fig. 6. In the first experiment (Fig. 6, circles), water was flown through the model string-type reactor with plastic strings $0.7 \mathrm{~mm}$ in diameter. As can be seen, the resulting displacement distribution closely resembles the histogram shown in Fig. 4, as expected. It is characterized by a large fraction of molecules traveling at relatively low aver-

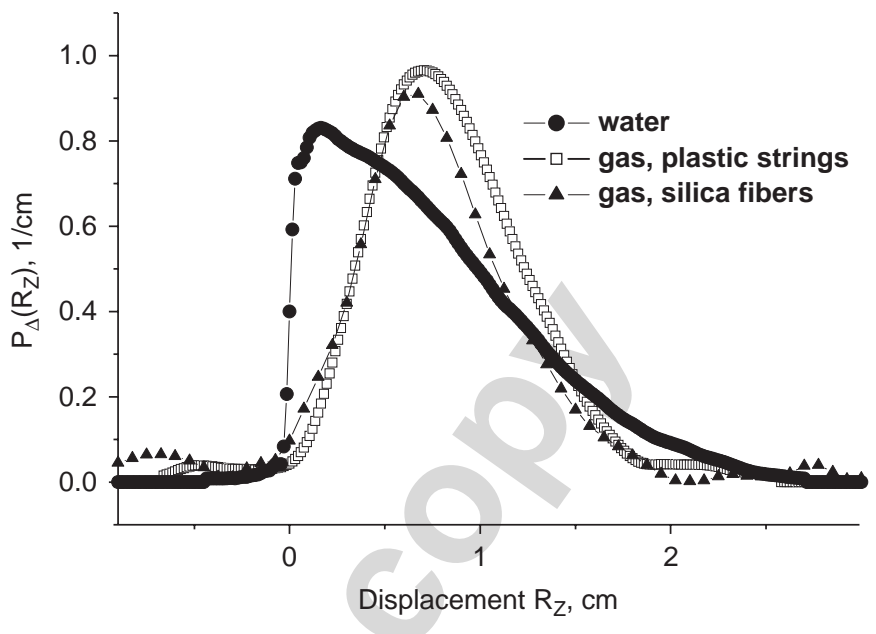

Fig. 6. Average propagators (displacement probability density) detected for water (circles) and gas (squares) in the model string-type reactor with $0.7 \mathrm{~mm}$ diameter plastic strings, and for gas in the real string reactor packed with silica fibers (triangles). The displacements during the encoding time of $\Delta=200 \mathrm{~ms}$ were measured in the direction of pressure gradient. Average flow velocities calculated from these displacement distributions are ca. $3.7 \mathrm{~cm} / \mathrm{s}$ for water and $4 \mathrm{~cm} / \mathrm{s}$ for gas in the model reactor, and $3.6 \mathrm{~cm} / \mathrm{s}$ for gas in the fiber-packed reactor.

age velocities. At the same time, the distribution of displacements of gas molecules in this reactor is quite different (Fig. 6, squares). In this case, there are no molecules with zero average velocity, and the shape of the propagator is somewhat closer to the desirable plug-flow behavior. An almost indistinguishable propagator is observed for the gas flow in the real string reactor packed with silica fibers (Fig. 6, triangles). Higher gas diffusivity values (smaller molecules, higher temperatures) should lead to a narrower propagator and to a flow pattern even closer to the plug-like flow. It is clear that the nature of plug-like flow in narrow channels is thus fundamentally different from that in porous media (e.g., in packed beds). In the latter case, the behavior of liquids and gases is similar, while in narrow channels the flow of a gas is very different from that of a liquid. It would be useful to compare the results obtained by MRM and PFG NMR techniques with the results of conventional tracer time-of-flight measurements.

\subsection{Tracer RTD studies}

The RTD of the compact string reactor was measured by the transient response method which is based on the monitoring of the reactor outlet signal when switching from the pure feed of inert gas to the one containing the tracer at the reactor inlet. The mean residence time in the reactor $(\tau)$ is calculated as a difference between the mean residence time in the set up with $\left(\tau_{S+R}\right)$ and without reactor $\left(\tau_{S}\right)$. Response curves $F(t)$ to the switch in concentration were measured at different flow rates. The dimensionless RTDs $E(\theta)$ were obtained as the derivatives of $F(t)$ with

$E(\theta)=E\left(t+\tau_{S}\right) \tau$ 


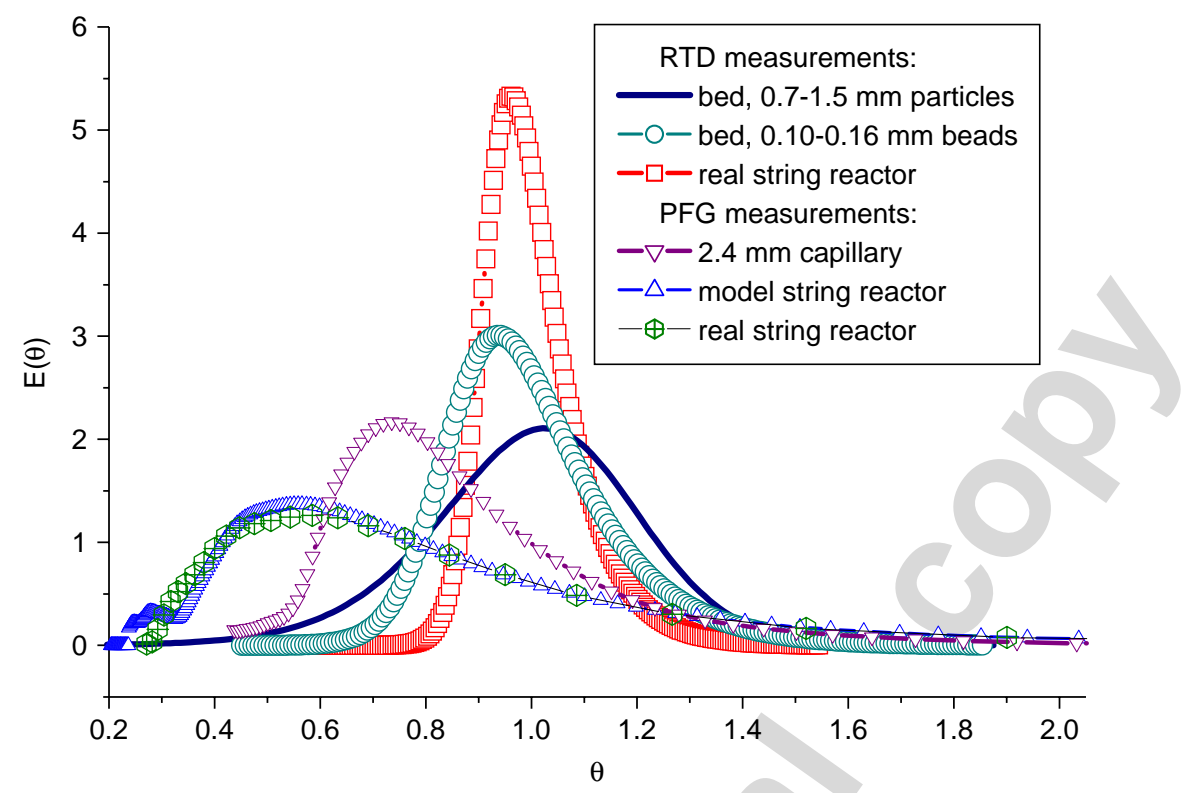

Fig. 7. Gas RTDs for the packed-bed reactors in comparison with the string reactor of the same dimensions measured with the transient response technique. The figure also shows the predicted gas RTDs calculated from the average propagators shown in Fig. 5 (capillary) and Fig. 6 (model string reactor and real string reactor packed with silica fibers).

and

$\theta=t / \tau$

The assumption was made that the RTD in the reactor was similar to the distributions for closed tubular reactors and can be described with the convection-dispersion equation:

$\frac{\partial C}{\partial t}=D_{\mathrm{ax}} \cdot \frac{\partial^{2} C}{\partial Z^{2}}-U \cdot \frac{\partial C}{\partial Z}=\frac{1}{\tau B o} \cdot \frac{\partial^{2} C}{\partial z^{2}}-\frac{\partial C}{\partial z} \cdot \frac{1}{\tau}$

with the Bodenstein number Bo defined as

$B o=\frac{U \cdot L}{D_{\mathrm{ax}}}$.

The $B o$ and $\tau$ values were optimized to obtain the best fit for the experimental data. The results of the RTD measurements in the string reactor packed with silica fibers and in the packedbed reactors are presented in Fig. 7 together with some of the PFG NMR data. The numerical characteristics of the RTDs measured by the transient response method for three different packings are summarized in Table 1.

Besides the significantly narrower RTD in the structured fiber-packed reactor $(B o=234)$ as compared to randomly packed beds, the pressure drop is also considerably lower. For the same volumetric gas flow rate of $30 \mathrm{~cm}^{3} / \min$ (STP) the pressure drop for the filamentous packing was found to be ca. $7 \mathrm{hPa}$. This value is roughly five times lower compared to the fixed bed of the same volume packed with $100-160 \mu \mathrm{m}$ diameter beads, and 3.5 times lower compared to reactors with the same mass of catalyst $(0.7-1.5 \mathrm{~mm}$ particles $)$.
Table 1

Variance of the RTD curves and Bo number values for the tracer transient response studies

\begin{tabular}{lcc}
\hline Bed type & Variance $\left(\sigma_{\theta}^{2}\right)$ & $\begin{array}{l}\text { Bodenstein } \\
\text { number }(\text { Bo })\end{array}$ \\
\hline $\begin{array}{l}\text { Randomly packed bed, irregular } \\
\text { particles }(0.7-1.5 \mathrm{~mm})\end{array}$ & $75 \times 10^{-3}$ & 30 \\
$\begin{array}{l}\text { Randomly packed bed, glass } \\
\text { beads }(0.10-0.16 \mathrm{~mm})\end{array}$ & $32 \times 10^{-3}$ & 66 \\
$\begin{array}{l}\text { Structured string-type reactor } \\
\text { with silica threads/fibers }\end{array}$ & $8.7 \times 10^{-3}$ & 234 \\
\hline
\end{tabular}

\subsection{Comparison of the results of PFG NMR and tracer RTD studies}

Quite often, a direct comparison of the results of two different techniques is not feasible, which is also true in our case. There are several reasons for that. Some of them are purely technical and can be overcome at least in principle. NMR and MRM experiments impose a number of restrictions on materials and samples that can be studied. For instance, metal-containing structures cannot be used, therefore the model devices studied here were fabricated from plastic parts. Besides, the two types of experiments utilize different gases. PFG NMR requires the use of gases with a reasonably high concentration of magnetic nuclei (e.g., ${ }^{1} \mathrm{H},{ }^{129} \mathrm{Xe},{ }^{3} \mathrm{He}$ ), but unlike tracer studies does not require the use of two different fluids.

More importantly, there is a big difference in what is measured with each technique. The time-of-flight technique measures tracer concentration at the outlet of a long reactor in response to a stepwise change of tracer concentration at the 
reactor inlet. As a result, the RTD obtained $(E(\theta))$ corresponds to the length scale of the entire reactor and reflects structural and transport properties averaged over the entire reactor length. In contrast, PFG NMR directly measures the displacements of molecules within a small section of a reactor $(3-4 \mathrm{~cm}$ in our case) for short observation times (200-300 $\mathrm{ms}$ in this work), i.e., for relatively small average displacements $(\leqslant 1 \mathrm{~cm})$. Therefore, any possible variations of structure and transport properties on longer length scales are not averaged.

In principle, it is possible to convert average propagators to RTDs mathematically according to the following equation:

$E(\theta)=E\left(C_{1} / R_{Z}\right)=C_{2} R_{Z}^{2} P_{\Delta}\left(R_{Z}\right)$.

The values of the two normalization constants, $C_{1}$ and $C_{2}$, are adjusted so that

$\int_{0}^{\infty} E(\theta) \mathrm{d} \theta=1$

and

$\int_{0}^{\infty} \theta E(\theta) \mathrm{d} \theta=1$

The RTDs calculated from the average propagators measured for butane gas and shown in Figs. 5 and 6 are presented in Fig. 7. They are obviously very poor approximations of the plug-flow-type behavior, with the RTD calculated for the single capillary ( $2.4 \mathrm{~mm}$ inside diameter) being somewhat better than the other two RTDs that correspond to the model string-type reactor and the real string reactor packed with silica fibers. It should be stressed, however, that these RTDs correspond to small average displacements $(0.7-0.8 \mathrm{~cm}$ for the string reactors, $1.05 \mathrm{~cm}$ for the capillary). Therefore, it can be expected that if it were possible to measure average propagators for the average displacement of ca. $20 \mathrm{~cm}$, the corresponding RTDs would have been much better. However, such an experiment is not feasible at present.

In the analysis of tracer time-of-flight results, an assumption is often made that transport in the reactor can be described with the convection-dispersion equation (3). The Bodenstein number $B o$ can be evaluated both from the experimentally measured RTD and from the average propagator, and thus could be used as a basis for comparison of the results obtained by different techniques. The $B o$ values evaluated from the average propagators are $B o=33$ for the capillary and $B o=8.2$ (silica fiber) and $B o=9$ (plastic) for the string reactors. If Eq. (3) could be applied to describe fluid flow, $B o$ values would be easy to correct for different lengths $L$ since $B o$ is proportional to $L$ (Eq. (4)). For instance, assuming that the observation time $\Delta$ is long enough to attain the long-time asymptote of dispersive flow, the single capillary $B o$ value for a $20 \mathrm{~cm}$ long reactor channel would be $B o=33 \times 20 / 1.05=630$. However, as mentioned above, this extrapolation of the $B o$ value relies on the applicability of Eq. (3). The latter implies that the average displacement of the fluid molecules increases linearly with time, while the width of the propagator increases as $t^{0.5}$, similar to characteristic diffusive displacements. As discussed above, this is expected to be the case for a single pipe, leading to the linear increase of $B o$ value with time or with the average travel distance. However, this is not expected to be the case for the model string-type reactor studied by PFG NMR in this work. This reactor comprises a large number of microchannels with very different areas of cross-section and thus with very different average velocities in each channel (cf. Fig. 3). The average propagator for the string-type system (Fig. 6) therefore is a superposition of a large number of individual propagators corresponding to the individual channels, with very different widths and centered at substantially different average displacements. If the observation time increases, each individual propagator will presumably broaden in proportion to $t^{0.5}$. At the same time, their relative positions will change with time too, and therefore the overall width of the envelope will increase with time faster than a square root of time dependence would imply. Similar behavior is expected for the string reactor packed with silica fibers, because the propagators for the two string reactors are very similar. Some small improvement in the behavior can still be expected with time, due to a better transverse averaging in the channels with the largest cross-sections. However, the overall improvement in RTD is expected to be significant only if inter-channel mixing becomes important.

\section{Discussion and conclusions}

In this work, the flow of water and butane gas in a single glass capillary and in string-type reactors was studied by magnetic resonance microimaging (MRM) and pulsed field gradient NMR (PFG NMR) techniques. The aim of the work was to find out what new information can be gained by applying the NMR-based techniques and to what extent it overlaps with and/or complements the results of time-of-flight measurements in terms of the evaluation of mass transport in microstructured reactors. It is demonstrated that the results obtained by the NMR/MRM techniques are difficult, if at all possible, to directly compare with the results obtained by more conventional techniques such as time-of-flight. In particular, the timeof-flight measurement averages mass transport over the length scale of the entire reactor, while MRM and PFG NMR measurements can be performed over length scales from microns to a few $\mathrm{cm}$. The two techniques also differ in the quantities that are measured. The conventional time-of-flight measurements yield RTDs, while MRM yields velocity maps and PFG NMR experiment provides probability density distribution of molecular displacements for a selected (and limited) time interval. The latter is equivalent to travel distance probability density function which in principle can be converted into an RTD, but it will still correspond to short equivalent reactor lengths of $1-2 \mathrm{~cm}$. Thus, such measurements characterize flow in a short section of a reactor. In general, extrapolation of these results to longer reactors cannot be performed reliably.

However, these differences do not necessarily represent a disadvantage. When transport in relatively long reactors is studied, the information obtained by MRM/NMR and more conventional techniques such as time-of-flight can be considered as complementary. While time-of-flight can characterize 
the overall performance of the reactor, MRM can reveal the reasons for the observed performance by providing access to the transport processes on shorter length scales inside the reactor. Furthermore, since MRM provides structural data along with transport information, it can be used to study structure-transport interrelation and to link the poor transport properties to the specific structural characteristics. In this work, the application of MRM technique to the characterization of the model stringtype reactor demonstrated that flow patterns in the channels with triangular cross-section formed by adjacent strings are highly unfavorable with respect to a plug-flow behavior. This is due to the low flow rates in the stretched corners of the cross-section, implying that for liquids such geometries should be avoided. At the same time, for gases, Taylor dispersion due to fast transverse diffusion can change the situation drastically if the equivalent channel diameter is small. However, if the packing is highly irregular, with broad variation of channels cross-sections, Taylor dispersion will result in a different average velocity in each individual channel. With little or no mixing between the channels, this will lead to a very broad RTD. Since the transient response method applied for the characterization of a real string reactor packed with silica fibers showed a narrow RTD $(B o=234)$, it can be concluded that in this string reactor the fibers in bundles are tortuous forming a network of not completely straight channels at large length scales. Therefore, an inter-channel mixing becomes significant leading to a much better performance on the length scale of the entire reactor. This example demonstrates the potential of MRM/NMR techniques to uncover structural and transport properties responsible for the observed overall reactor performance.

At the same time, MRM and PFG NMR techniques can be invaluable when studying microreactors characterized by short reactor lengths. In such cases, the length of the entrance and exit sections are comparable to the size of the reactor itself and the inflow and outflow effects cannot be ignored. Since MRM and PFG NMR techniques do not use tracers and the measurements can be performed locally, such measurements will not suffer from entry and exit effects. Furthermore, such effects can be characterized, if necessary. PFG NMR can be combined with MRM to measure average propagators with sub-millimeter spatial resolution in order to gain access to the transport behavior in microreactors. Besides, the modern MRM/NMR toolkit has the necessary instruments to address the issue of the inter-channel mixing directly, using the number of existing approaches which can correlate displacements of molecules along different axes and/or unravel the exchange processes in the coordinate or velocity space (Callaghan and Manz, 1994; Stapf et al., 1998; Han et al., 2000; Blumich et al., 2001; Khrapitchev et al., 2002; Han and Stapf, 2006). All this can lead to the development of a new research field-the NMR microimaging of microreactors.

\section{Notation}

$B o=U L / D_{\mathrm{ax}} \quad$ Bodenstein number

$C \quad$ tracer concentration

$\begin{array}{ll}d_{\mathrm{f}} & \text { equivalent channel diameter } \\ D_{\mathrm{ax}} & \text { axial dispersion coefficient } \\ E(\theta) & \text { residence time distribution function } \\ L & \text { reactor length } \\ P_{\Delta}\left(R_{Z}\right) & \text { probability density function (distribution } \\ & \text { of displacements } R_{Z} \text { during time interval } \\ & \Delta), 1 / \mathrm{cm} \\ P_{\Delta}\left(V_{Z}\right) & \text { probability density function (distribution } \\ & \left.\text { of velocities } V_{Z}\right), \text { s/cm } \\ R_{Z}=Z-Z_{0} & \text { axial displacement } \\ t & \text { time } \\ U & \text { mean axial flow velocity } \\ V_{Z} & \text { axial flow velocity } \\ Z=Z / L & \text { dimensionless axial coordinate } \\ Z & \text { axial coordinate }\end{array}$

\section{Greek letters}

$\Delta=t-t_{0}$

$\theta=t / \tau$

$\sigma_{\theta}^{2}$

$\tau=L / U$

$\tau_{S}$

$\tau_{S+R}$

displacement encoding time interval (in a PFG NMR experiment)

dimensionless time

variance of the RTD curves obtained by tracer transient response technique mean residence time mean residence time without the reactor in transient response measurements mean residence time with the reactor in transient response measurements

\section{Acknowledgments}

This work was supported by RFBR (05-03-32472a), RFBRNWO (03-03-89014-NWO, 047.015.006), SB RAS (integration Grant \#11), RAS (5.1.1 and 5.2.3), and the President's program of support of leading scientific schools (NSch-4821.2006.3). I.V. Koptyug thanks the Russian Science Support Foundation for financial support.

\section{References}

Alépée, C., et al., 2000. Fast heating and cooling for high temperature chemical microreactors. In: Ehrfeld, W. (Ed.), Proceedings of the Fourth International Conference on Microreaction Engineering. Springer, Berlin, p. 514.

Amin, M.H.G., et al., 1997. Study of flow and hydrodynamic dispersion in a porous medium using pulsed-field-gradient magnetic resonance. Proceedings of the Royal Society of London, Series A, Mathematical and Physical Sciences 453, 489-513.

Blumich, B., et al., 2001. Two-dimensional NMR of velocity exchange: VEXSY and SERPENT. Journal of Magnetic Resonance 152, 162-167.

Callaghan, P.T., 1991. Principles of Nuclear Magnetic Resonance Microscopy. Clarendon Press, Oxford.

Callaghan, P.T., Manz, B., 1994. Velocity exchange spectroscopy. Journal of Magnetic Resonance A 106, 260-265.

Ehrfeld, W., Hessel, V., Lowe, H., 2000. Microreactors: New Technology for Modern Chemistry. Wiley, New York.

Fukushima, E., 1999. Nuclear magnetic resonance as a tool to study flow. Annual Review of Fluid Mechanics 31, 95-123.

Gladden, L.F., 2003. Magnetic resonance: ongoing and future role in chemical engineering research. A.I.Ch.E. Journal 49, 2-9. 
Gladden, L.F., et al., 2002. Magnetic resonance imaging of single- and two-phase flow in fixed-bed reactors. Applied Magnetic Resonance 22, 201-212.

Goetz, J., et al., 2002. Visualisation of flow processes in packed beds with NMR imaging: determination of the local porosity, velocity vector and local dispersion coefficients. Chemical Engineering and Processing 41, 611-629.

Han, S., Blumich, B., 2000. Two-dimensional representation of position, velocity and acceleration by PFG-NMR. Applied Magnetic Resonance 18, $101-114$.

Han, S., Stapf, S., 2006. Fluid flow and trans-membrane exchange in a hemodialyzer module. In: Stapf, S., Han, S. (Eds.), NMR Imaging in Chemical Engineering. Wiley-VCH, Weinheim, pp. 457-470.

Han, S., Stapf, S., Blumich, B., 2000. Two-dimensional PFG NMR for encoding correlation of position, velocity, and acceleration in fluid transport. Journal of Magnetic Resonance 146, 169-180.

Han, S., Granwehr, J., Hilty, C., 2006. Broadening the application range of NMR and MRI by remote detection. In: Stapf, S., Han, S. (Eds.), NMR Imaging in Chemical Engineering. Wiley-VCH, Weinheim, pp. 139-163.

Heath, C.A., et al., 1990. Magnetic resonance imaging and modeling of flow in hollow-fiber bioreactors. A.I.Ch.E. Journal 36, 547-558.

Hilty, C., et al., 2005. Microfluidic gas-flow profiling using remote-detection NMR. Proceedings of the National Academy of Sciences of the USA 102, 14960-14963.

Horny, C., et al., 2004. Micro-structured string-reactor for autothermal production of hydrogen. Chemical Engineering Journal 101, 3-9.

Khrapitchev, A.A., et al., 2002. Spectrally resolved velocity exchange spectroscopy of two-phase flow. Journal of Magnetic Resonance 159 , 36-45.

Kiwi-Minsker, L., Renken, A., 2005. Microstructured reactors for catalytic reactions. Catalysis Today 110, 2-14.

Koptyug, I.V., Sagdeev, R.Z., 2002. Applications of NMR tomography to mass transfer studies. Russian Chemical Reviews 71, 789-835.

Koptyug, I.V., et al., 2000. Thermally polarized ${ }^{1} \mathrm{H}$ NMR microimaging studies of liquid and gas flow in monolithic catalysts. Journal of Magnetic Resonance 147, 36-42.
Koptyug, I.V., et al., 2002. NMR studies of hydrocarbon gas flow and dispersion. Applied Magnetic Resonance 22, 187-200.

Laukemper-Ostendorf, S., et al., 1998. NMR imaging of flow in hollow fiber hemodialyzers. Journal of Membrane Science 138, 287-295.

Lebon, L., Leblond, J., 2002. PFG NMR measurements of flow through porous media: effects of spatial correlations of the magnetic field and the velocity field. Journal of Magnetic Resonance 159, 13-24.

Lebon, L., et al., 1996. Pulsed gradient NMR measurements and numerical simulation of flow velocity distribution in sphere packings. Physics of Fluids 8, 293-301.

Lebon, L., et al., 1997. Experimental measurement of dispersion processes at short times using a pulsed field gradient NMR technique. Physics of Fluids 9, 481-490.

Mansfield, P., Bencsik, M., 1998. Fluid flow in porous systems. Magnetic Resonance Imaging 16, 451-454.

Packer, K.J., Tessier, J.J., 1996. The characterization of fluid transport in a porous solid by pulsed gradient stimulated echo NMR. Molecular Physics 87, 267-272.

Seymour, J.D., Callaghan, P.T., 1997. Generalized approach to NMR analysis of flow and dispersion in porous media. A.I.Ch.E. Journal 43, 2096-2111.

Stapf, S., et al., 1998. Spatial correlations and dispersion for fluid transport through packed glass beads studied by pulsed field-gradient NMR. Physical Review E 58, 6206-6221.

Tallarek, U., et al., 1998. Study of transport phenomena in chromatographic columns by pulsed field gradient NMR. Journal of Physical Chemistry B 102, 3486-3497.

Taylor, G.I., 1953. Dispersion of soluble matter in solvent flowing slowly through a tube. Proceedings of the Royal Society of London, Series A, Mathematical and Physical Sciences 219, 186-203.

Tessier, J.J., et al., 1997. NMR measurements and numerical simulation of fluid transport in porous solids. A.I.Ch.E. Journal 43, 1653-1661.

Watson, A.T., et al., 2002. NMR determination of porous media property distributions. Annual Reports on NMR Spectroscopy 48, 113-144.

Wolfrath, O., et al., 2001. Novel membrane reactor with filamentous catalytic bed for propane dehydrogenation. Industrial and Engineering Chemistry Research 40, 5234-5239. 